\title{
Critical contribution of 3' non-seed base pairing to the in vivo function of the evolutionarily conserved let-7a microRNA
}

Ye Duan ${ }^{1}$, Isana Veksler-Lublinsky², Victor Ambros ${ }^{1,{ }^{*}}$

${ }^{1}$ Program in Molecular Medicine, University of Massachusetts Medical School, Worcester, MA, 01605, USA

${ }^{2}$ Department of Software and Information Systems Engineering, Ben-Gurion University of the Negev, Beer-Sheva, 8410501, Israel

*Corresponding author: victor.ambros@umassmed.edu

Running Title: 3' non-seed region is critical for let-7a in vivo function

Key Words: microRNA, let-7, lin-41, let-7 family, microRNA seed, microRNA non-seed, CRISPR, post-transcriptional regulation, daf-12, $h b l-1$ 


\section{Abstract:}

2 MicroRNAs are endogenous regulatory non-coding RNAs that exist in all multi-cellular

3 organisms. Base-pairing of the seed region (g2-g8) is essential for microRNA targeting, however,

4 the in vivo functions of the 3' non-seed region (g9-g22) are less well understood. Here we report

5 the first systematic investigation of the in vivo roles of 3' non-seed nucleotides in microRNA let-

67 a, whose entire g9-g22 region is conserved among bilaterians. We found that the 3' non-seed

7 sequence functionally distinguishes let-7a from its family paralogs. The complete pairing of $\mathrm{g} 11$ -

$8 \mathrm{~g} 16$ is essential for let-7a to fully repress multiple key targets, including evolutionarily conserved

9 lin-41, daf-12 and hbl-1. Nucleotides at g17-g22 are less critical but may compensate for

10 mismatches in the g11-g16 region. Interestingly, the 3' non-seed pairing of let-7a can be critically

11 required even with sites that permit perfect seed pairing. These results provide evidence that the

12 specific configurations of both seed and 3' non-seed base-pairing can critically influence

13 microRNA function in vivo. 


\section{Introduction}

MicroRNAs (miRNA) are short non-coding RNAs that exist in all metazoans (Friedman et al.,

16 2009; Lee et al., 1993; Nelson and Ambros, 2021). Mature miRNAs are bound by Argonaute

17 proteins (AGO) to form the miRNA-induced silencing complex (miRISC), and base pair with complementary sites in the 3' untranslated region (UTR) of target RNAs. miRISC binding leads to post-transcriptional repression of target gene expression through translational inhibition and/or target RNA destabilization (Bartel, 2018). miRNAs are critical for the regulation of diverse physiological processes across species (Ambros, 2004; Bartel, 2018). Up to 60\% of human genes are estimated to be regulated by miRNAs, and disfunction of miRNAs is implicated in multiple human diseases (Friedman et al., 2009; Paul et al., 2018).

The miRNA seed corresponds to the 6-7 contiguous nucleotides beginning at the second nucleotide (g2) from 5' end and is understood to be the dominant determinant of miRNA targeting efficacy and specificity. Structural and biochemical studies indicate that miRNA seed, especially g2-g5, is critical for target recognition and binding (Salomon et al., 2015; Schirle et al., 2014). Complementarity to the miRNA seed is the most evolutionarily conserved feature of miRNA target sites, and genetically disrupting seed complementarity can result in de-repression of miRNA targets (Lai, 2002; Lewis et al., 2005; Lim et al., 2005).

Organisms commonly contain multiple genes encoding miRNAs with identical seed sequences, which are grouped into seed families. miRNAs of the same family can in principle recognize shared targets through seed pairing and thereby function redundantly (Abbott et al.,

34 2005; Alvarez-Saavedra and Horvitz, 2010; Brenner et al., 2012). Meanwhile, miRNAs with 35 identical seed but divergent 3' non-seed nucleotides (g9-g22) can exhibit target site selectivity 36 driven by the extent of non-seed base pairing (Broughton et al., 2016; Wahlquist et al., 2014). 
The let-7 (lethal-7) family miRNAs are distributed widely across the bilaterians, consistent with evolutionary origins in a bilaterian ancestor (Hertel et al., 2012; Wolter et al., 2017). Similarities in the developmental profile of let-7 family miRNAs across diverse species and genetic analysis in model organisms suggest evolutionary conservation of let-7a family functionality

41 (Roush and Slack, 2008; Tennessen and Thummel, 2008). In nematodes, let-7 family miRNAs

42 function in the heterochronic gene pathway to promote the stage-specific cell fate transitions

43 during larval development (Abbott et al., 2005; Reinhart et al., 2000). Similarly, let-7 family

44 miRNAs control cellular transitions from pluripotency to differentiation and function in tumor

45 suppression in mammals, and the timing of adult fates during metamorphosis in insects (Balzeau et al., 2017; Lee et al., 2016; Sokol et al., 2008).

Notably, almost all bilaterian genomes encode at least one let-7 family isoform (let-7a), in which all nucleotides in the 3' non-seed region are highly conserved (Fig. S1A) (Hertel et al., 2012; Pasquinelli et al., 2003). This deep conservation suggests that the 3 ' non-seed region may also be associated with essential functions which evolutionarily constrained the sequence. Indeed,

51 high-throughput analyses suggested that miRNA 3' non-seed regions can contribute substantially 52 to miRNA-target interactions (Grosswendt et al., 2014; Helwak et al., 2013). Pairing to the 3' non53 seed region, especially g13-g16, is thought to enhance target repression in certain contexts 54 (Brennecke et al., 2005; Grimson et al., 2007). Recent structural studies of human AGO 55 complexed with miRNA and target also revealed that g13-g16 can form an A-form helix with the 56 target within miRISC, and can thereby increase miRISC-target affinity and specificity (Sheu57 Gruttadauria et al., 2019b; Xiao and MacRae, 2020). Notably, some miRNA target sites have 58 imperfect complementarity with the miRNA seed, such as GU base pair or nucleotide bulge, and 59 are accompanied by extensive non-seed pairing (Chi et al., 2012; Grimson et al., 2007; Vella et 60 al., 2004). In such cases, the 3' non-seed pairing is thought to compensate for the imperfect seed 61 pairing and this compensatory configuration can determine the target specificity among family 
62 isoforms (Brancati and Grosshans, 2018; Brennecke et al., 2005; Doench and Sharp, 2004).

63 However, the relative contributions of specific 3' non-seed nucleotides to the in vivo function of

64 miRNAs remain unclear.

Here, we report a systematic investigation of how the 3' non-seed nucleotides contribute to

66 the in vivo function of the evolutionarily conserved let-7a. We employ CRISPR/Cas9 genome

67 editing of $C$. elegans to systematically introduce defined mutations into the let-7a and its

68 conserved target lin-41. We show that the sequence of g11-g16 is essential for let-7a in vivo

69 function, whilst g17-g22 pairing is less critical than g11-g16 pairing, but can partially compensate

70 for mismatches in $911-g 16$. We confirm that the 3' non-seed sequence of let-7a confers specificity

71 relative to its family paralogs. Furthermore, we show that lin-41, as well as heterochronic genes

72 daf-12 and $h b l-1$, are let-7a targets that require 3' non-seed pairing, and that proper repression of

73 lin-41 requires complementarity to both the seed and critical 3' non-seed region of let-7a.

74 Strikingly, the 3' non-seed pairing is functionally required even when the lin-41 3' UTR was

75 mutated to accommodate perfect seed pairing, suggesting that the g11-g16 of let-7a engage in

76 essential interactions that, in parallel with seed pairing, influence miRISC repressing activity.

\section{Results}

The 3' non-seed sequence is essential for the in vivo functional specificity of let-7a compared to paralogs.

An examination of the phylogenetic distribution of let-7 family miRNA genes (Fig. 1A, S1B)

81 emphasizes the presence of let-7 family in most bilaterians with available miRNA annotations in

82 miRbase 22.1 (Kozomara et al., 2019). 91.5\% $(n=117)$ of bilaterian species have at least one let-

837 family isoform with no more than 3 different nucleotides from let-7a (Fig. S1C-E). Alignment of

84 the isoforms closest to let-7a from each of the 117 bilaterian species indicates that all the g9-g22

85 nucleotides exhibit conservation ranging from $86 \%$ to $99 \%$, and $75.2 \%$ of the 117 species contain 
a let-7 miRNA completely identical to let-7a (Fig. 1B, S1F). This degree of conservation is not observed for other conserved miRNAs (Fig. S1G-H). The deep conservation of let-7a suggests that let-7a is associated with essential functions that depend on the identity of the 3' non-seed nucleotides.

To test the hypothesis that the 3' non-seed nucleotides are critical for let-7a in vivo function, we used CRISPR/Cas9 to mutate the endogenous let-7a in C. elegans by swapping its 3' nonseed sequence with the sequence of its closest paralog miR-84 (Fig. 2A). In the resulting let7(ma341) mutant, the passenger strand was also mutated to preserve the precursor miRNA (premiRNA) structure. The temporal expression profile of miR-84 miRNA in let-7(ma341) is consistent with expression from the endogenous mir-84 locus (starting in L2) combined with expression from the edited let-7 locus (peaking in L4) (Fig. 2B-C). Moreover, in the mir-84(n4037, null) background, miR-84 expression from the let-7(ma341) locus alone exhibits a temporal profile similar to that of normal let-7a (Fig. 2D-E). Note that the detection method stringently distinguishes let-7a from miR-84, as let-7a was not detected in let-7(ma341) animals and miR-84 was not detected in mir84(n4037) animals (Fig. 2C-E). These results confirm that let-7(ma341) expresses miR-84 miRNA 101 instead of let-7a.

In wild-type (WT) animals, the seam cells (hypodermal stem cells) go through asymmetric divisions at each larval stage as one daughter cell differentiates to Hyp7 cells (hypodermis) while

104 the other remains stem cell (Sulston et al., 1983). At L4 molt, the seam cells exit the cell cycle, fuse, and produce the adult-specific cuticle structure referred to as adult alae. In let-7(ma341), we found that seam cells underwent an extra round of cell division after L4 molt, resulting in extra seam cells and incomplete alae in young adults (Fig. 2F-G, Table S1), and let-7(ma341) adults exhibited impaired expression of the adult-specific hypodermal reporter COL-19::GFP (Fig. 2H-I).

109 These results indicate that let-7(ma341) causes retarded heterochronic phenotypes. let-7(ma341) 110 also exhibits lethality as a consequence of bursting at the vulva (Fig. 2J-L). The lethality and 
111 retarded phenotypes are consistent with let-7a loss-of-function (If) phenotypes (Reinhart et al.,

112 2000), indicating that the in vivo function of let-7a cannot be substituted by its closest paralog

113 miR-84, even when miR-84 is expressed in a developmental profile essentially identical to let-7a.

114 Since let-7a and mir-84 share the seed sequence, these findings indicate that the 3' non-seed

115 sequence is a critical determinant of the in vivo function and specificity of let-7a.

\section{Nucleotides 11-16 are each functionally essential for let-7a in vivo.}

We next sought to characterize how each 3' non-seed nucleotide contributes to in vivo function of let-7a. We performed a single nucleotide mutational screen of the let-7a non-seed region using the "jump-board" strategy (Duan et al., 2020a). For each g9-g22 nucleotide, we mutated both the miRNA guide and the passenger strands to preserve the pre-miRNA structure (Fig. 3A, S2A). To confirm the expression of the mutant miRNAs, we performed small RNA sequencing of all the mutants and found that the abundance of the mutated let-7a miRNA in each mutant was similar to that of the native let-7a in WT, with a variance $(<10 \%)$ that we judge not

124 sufficient to cause If phenotypes by reduced expression (Fig. 3B). Note that the strains with 125 mutations at g11-g16 also contained a WT let-7 allele on a genetic balancer umnls25(mnDp1), 126 hence both WT and mutant let-7a were expressed. characteristic of let-7(If), leading to lethality and dramatically reduced numbers of progeny, quantitatively similar to let-7(null) (Fig. 3C-D). Single nucleotide mutations at g11-g13 also resulted in impaired expression of COL-19::GFP in Hyp7 cells, and adult alae morphogenesis

131 defects, indicative of retarded heterochronic phenotypes characteristic of severe let-7(If) (Fig. 3C, 132 S2B, Table S1). Single nucleotide mutations at g14-g16 also resulted in phenotypes distinctly 133 characteristic of let-7(If), although milder than g11-g13 mutations (Fig. 3C-E). By contrast, single 134 nucleotide mutations at g9-g10 or g17-g22 did not cause visible phenotypes (Fig. 3C, F, Table 
135 S1). The mutational screen reveals g11-g16 as a critical non-seed region of let-7a, with g11-g13 136 somewhat more critical than g14-g16 (Fig. 3G).

137 Nucleotides beyond g16 can compensate for mismatches in the critical non-seed region.

We found that mutating the g18 of let-7a into any other three nucleotides did not result in If 139 phenotypes (Fig. S2C). Moreover, simultaneous mutations of g17-g19, g20-g22, or g17-g22 did 140 not cause apparent If phenotypes, even with sensitized conditions like non-physiological 141 temperatures or pathogen-containing cultures (Fig. S2C and data not shown). The insignificance 142 of $\mathrm{g} 17-\mathrm{g} 22$ nucleotides in the screen stands out in contrast with the deep conservation of the 143 entire let-7a sequence, especially with g18 as the second most conserved nucleotide (98.3\%). 144 We thus hypothesized that g17-g22 may contribute to target repression in cases where 145 mismatches occur within the g11-g16 region.

146 To test this possibility, we used the let-7(ma449, U16G) mutation as a genetically sensitized 147 background with moderate phenotypes, and generated a compound mutant let-7(ma449ma435) 148 with both U16G and U18A mutations. We observed that let-7(ma449ma435) exhibited a strong 149 vulva bursting phenotype and retarded adult alae (Fig. 3H, S2C-D), which are significantly more 150 penetrant than that of each single mutant. We suggest that $\mathrm{g} 18$, and by implication other g17-g22 151 nucleotides, may contribute to interactions that involve mismatches in the g14-g16 sub-critical 152 region.

It is also possible that g17-g22 pairing could be critical for target repression associated with 154 phenotypes that we have not measured here. To investigate this possibility, we assessed 155 molecular phenotypes of let-7(ma435) by ribosome profiling and RNA-seq (Fig. S3, Table S2).

156 We found that the expression levels of multiple genes are significantly changed by the U18A 157 mutation, both translationally and transcriptionally, supporting the hypothesis that there are in vivo 158 circumstances where let-7a g17-g22 nucleotides are critical for proper target regulation. 
Evolutionarily conserved NHL gene lin-41/Trim71 is a direct target of let-7a across species, and repression of lin-41/Trim71 by let-7a is essential for normal development in both invertebrates and vertebrates (Ecsedi and Grosshans, 2013; Worringer et al., 2014). In C. elegans, robust repression of lin-41 by let-7a is critical for the L4-to-adult progression, and mutations disrupting the seed pairing between let-7a and its complementary sites (LCSs) in the lin-41 3' UTR result in reiteration of L4 cell fates and severe vulva defects (Aeschimann et al., 2019). Notably, neither of the two lin-41 LCSs have perfect seed complementarity with let-7a due to a GU pair and a target A-bulge, respectively, while both LCSs contain complementarity to let-7a g11-g19, suggesting that 3' pairing may be essential to compensate for the weak seed pairing in this context (Fig. 4A).

We sought to verify whether lin-41 is de-repressed in the let-7a critical non-seed mutants and whether the de-repression causes the If phenotypes. We used the U13A mutant to represent the

171 critical non-seed nucleotides due to its severe phenotypes and the high conservation at g13. At

172 late L4 stage of WT, endogenously tagged GFP::LIN-41 is undetectable in hypodermal cells due

173 to let-7a repression (Fig. S4A) (Spike et al., 2014). In contrast, in let-7(ma432, U13A), we

174 observed elevated peri-nucleolus expression of GFP::LIN-41 in seam and Hyp7 cells, suggesting

175 de-repression of lin-41 (Fig. S4B). To confirm that the lin-41 de-repression in let-7(ma432) results

176 specifically from disruption of g13 pairing, we restored the native pairing configurations between

177 let-7(ma432) and lin-41 by introducing compensatory mutations at both LCSs in lin-41 3' UTR

178 (ma480) (Fig. 4A). We did not detect the abnormal expression of GFP::LIN-41 in lin-

179 41(ma480);let-7(ma432), indicating the restoration of lin-41 repression by re-establishing the WT

180 3' non-seed pairing configuration (Fig. S4C). We found that in lin-41(ma480);let-7(ma432), the If

181 phenotypes caused by ma432 mutation were substantially rescued based on reduced lethality, 182 increased number of progeny and normal adult alae (Fig. 4B-D, F, Table S1). The rescue of let- 
7(ma432) phenotypes by restoring $g 13$ pairing indicates that de-repression of lin-41 is the major contributor to the If phenotypes of let-7a critical non-seed mutants.

In parallel, we found that when present with WT let-7a, lin-41(ma480) exhibited elevated perinucleus expression of GFP::LIN-41 in L4 stage, suggesting that disrupting the critical non-seed pairing by mutations in the target sites also causes lin-41 de-repression (Fig. S3D). As expected, we also observed vulva integrity defects and retarded heterochronic phenotypes in lin-41(ma480) (Fig. 4E-F, Table S1), supporting the conclusion that 3' non-seed pairing between let-7a and lin41 is essential for maintaining the robust repression of lin-41.

Disruption of $3^{\prime}$ non-seed pairing of let-7a results in de-repression of additional targets including daf-12 and $h b l-1$.

Although lin-41(ma480);let-7(ma432) showed alleviation of the If phenotypes from let7(ma432), the double mutant is nevertheless not completely WT and exhibits residual defects in vulva morphogenesis and egg-laying capacity (Fig. 4D, F). Also, COL-19::GFP expression in Hyp7 cells of lin-41(ma480);let-7(ma432) adults is reduced at $25^{\circ} \mathrm{C}$, indicating residual retarded phenotypes (Fig. 4G). These residual phenotypes suggest that although lin-41(ma480);let7(ma432) restores the lin-41 repression, additional let-7a targets may be de-repressed by the non-seed mutation (Fig. 4H). We refer to this proposed class of let-7a targets that require critical non-seed pairing as " 3 '-sup targets". Consistent with the reasoning that the phenotypes of let7(ma432) result from the over-expression of both lin-41 and other 3'-sup targets, the lin-41(ma480) mutant, where only lin-41 is over-expressed since the mutation is on the target, exhibits weaker phenotypes than let-7(ma432) (Fig. 4F, Table S1).

To identify the additional let-7a 3'-sup targets, we screened for genes that are de-repressed by the let-7a critical non-seed mutation. Accordingly, we compared gene expression between WT 206 and lin-41(ma480);let-7(ma432) using ribosomal profiling (Ribo-seq), which assesses gene 
expression on translational level (Ingolia, 2016). We used lin-41(ma480);let-7(ma432) to exclude changes in lin-41 downstream genes. We found that at L4 stage, 135 genes were significantly over-expressed $>2$ fold and 351 genes were significantly over-expressed $>1.5$ fold in lin41(ma480);let-7(ma432) compared to WT (Fig. 5A, Table S3). The translational levels of lin-41

211 and its direct downstream genes were not significantly changed, indicating that the de-repressed 212 genes in lin-41(ma480);let-7(ma432) are independent of lin-41 pathway (Fig. S6A) (Aeschimann 213 et al., 2019).

We next sought to identify the let-7a 3'-sup targets among the over-expressed genes by identifying LCSs in the 3' UTRs. We developed a computational approach to predict let-7a sites involving both seed and critical non-seed complementarity based on the method described in

217 (Veksler-Lublinsky et al., 2010). We firstly identified candidate sites based on seed complementarity to let-7a, allowing for both perfect and imperfect pairing at $\mathrm{g} 2 \mathrm{~g} \mathrm{~g}$, and then extended these sites to check whether they also included at least 3 consecutive base pairs in g11-g16, among which g13 must be paired. We identified 624 genes whose 3' UTR contain let7a 3'-sup sites in C. elegans, among which 8 genes are over-expressed in the g13 mutant (Fig. 5A, Table S4). We reasoned that these 8 genes could include let-7a 3'-sup targets whose derepression contributes to the residual phenotypes lin-41(ma480);let-7(ma432).

The heterochronic genes daf-12 and hbl-1 were among the 8 candidate 3'-sup targets; and the 3' UTRs of both genes contain multiple let-7a sites with critical non-seed pairing (Fig. 5A, S6BD). We thus suggest that the WT L4-to-adult cell fate transitions require 3' non-seed pairing of let-

227 7a to repress not only lin-41, but also daf-12 and $h b l-1$, and accordingly the daf-12 and $h b l-1$ de228 repression contributes to the residual retarded phenotypes of lin-41(ma480);let-7(ma432). 229 Constant with this supposition, we found that knocking down either daf-12 or hbl-1 by RNAi could 230 rescue the abnormal COL-19::GFP pattern in lin-41(ma480);let-7(ma432) (Fig. 5B). Interestingly, 231 whereas restoring lin-41 repression alone could rescue the abnormal adult alae phenotype of let- 
232 7(ma432) (Table S1), rescuing the reduced COL-19::GFP in Hyp7 requires restoring repression 233 daf-12 or $h b l-1$ in addition to lin-41 (Fig. 5B). This suggests that the L4 repression of lin-41 by let$2347 a$ is sufficient to regulate adult alae formation, while the robust adult-specific differentiation of 235 Hyp7 requires the repression of $h b l-1$, daf-12 and lin-41.

236 Repression of let-7a 3'-sup targets can involve translational suppression and/or mRNA decay.

miRNA-mediated post-transcriptional regulation includes translational repression and/or mRNA destabilization, indicated by decreased translational efficiency (TE) and reduced mRNA abundance (Bazzini et al., 2012; Giraldez et al., 2006). It has been shown that let-7a can regulate

241 lin-41 through a combination of both modes (Bagga et al., 2005; Nottrott et al., 2006). To assess

242 the target repression mechanisms associated with let-7a 3' non-seed region, we analyzed our 243 Ribo-seq data of lin-41(m480);let-7(ma432) in combination with RNA-seq (Fig. 5C, S7, Table S3).

244 Previous reports showed that miRNAs may cause deadenylation before mRNA decay, thus

245 RNAseq libraries from poly (A)-enriched mRNA may potentially have a bias against deadenylated 246 but temporally stable mRNA (Djuranovic et al., 2012; Wu et al., 2006). We thus used ribosomal 247 RNA depletion with anti-sense oligos specifically designed for C.elegans to enrich the mRNA 248 (Duan et al., 2020b). We found that the set of de-repressed let-7a 3'-sup targets in lin249 41(ma480);let-7(ma432) include examples of significantly increased mRNA abundance (i.e., grd250 10) or TE (i.e., seld-1, hbl-1), or both modes (i.e., daf-12), (Fig. 5C). Thus, target recognition 251 involving the let-7a non-seed region can elicit inhibition of either TE or mRNA stability, or both, 252 likely depending on context. 
41.

The involvement of 3' non-seed pairing to compensate for imperfect seed pairing has been observed for certain miRNA-target interactions in various systems (Bartel, 2018). However, it remains unclear whether miRISC may elicit other seed-independent functions that critically require non-seed pairing. We anticipated that if 3' non-seed pairing were only required in the context of weak seed pairing, configuring miRNA sites for full seed complementarity should render mutations in the lin-41 3' UTR designed to produce various configurations of seed and non-seed pairing with let-7a (Fig. 6). Importantly, all the configurations were constructed by mutating both result from de-repression of lin-41 without confounding effects from other let-7a targets.

As noted above, lin-41(ma480), which carries g13:t13 mismatch to WT let-7a, exhibited strong retarded heterochronic phenotypes and vulva integrity defects due to lin-41 de-repression (Fig. 6E). To test whether the g13:t13 mismatch is deleterious only in the context of imperfect seed pairing, we mutated the LCSs of lin-41(ma480) to repair the A-bulge and GU pair in the seed pairing. The resulting lin-41(ma501ma480) compound mutant is expected to enable consecutive

270 g2-g8 Watson-Crick pairing to let-7a, whilst maintaining the g13:t13 mismatch (Fig. 6B). We found 271 that lin-41(ma501ma480) animals exhibited a substantial rescue of the If phenotypes from lin272 41(ma480) (Fig. 6B), confirming the essential compensatory effect of let-7a non-seed pairing to imperfect seed complementarity.

275 rescue of retarded COL-19::GFP expression was incomplete (11\% retarded, Fig. 6B), suggesting

276 that enabling perfect seed pairing of let-7a was insufficient to fully compensate for the g13:t13 277 mismatch, and thereby a non-seed mismatch can be deleterious even in the context of perfect 
seed pairing. To further explore the requirements for non-seed pairing in the context of perfect seed complementarity, we combined ma501 with a compound mutation (ma545) to mismatch g11-g13 of let-7a (Fig. 6C). The LCSs in lin-41(ma501ma545) were designed to not permit nonseed pairing with let-7a paralogs. Interestingly, we found that lin-41(ma501ma545) exhibited strong vulva integrity defects and retarded COL-19::GFP expression, suggesting that lin-41 was de-repressed despite the perfect seed pairing to let-7a (Fig. 6C). The loss of let-7a regulation in lin-41(ma501ma545) indicates that even with perfect seed complementarity, the 3' non-seed pairing still contributes critically to the functional targeting of lin-41.

Meanwhile, the full complementarity to let-7a seed in lin-41(ma501) should in principle enable let-7a family paralogs to repress lin-41, assuming that perfect seed pairing alone was sufficient to confer full repressing efficacy (Fig. 7A). Since the expression levels of let-7a family paralogs miR48, miR-84 and miR-241 combined have been shown to exceed the level of let-7a at L4 stage in pairing to lin-41(ma501) by let-7a paralogs might be able to robustly repress lin-41 in the absence of let-7a (Fig. 7C). Strikingly, lin-41(ma501);let-7a(ma393, null) only exhibited limited reduction of

293 the severe vulva integrity defects of let-7a(ma393), indicating that the let-7a family paralogs only 294 render a minor degree of lin-41 repression with the engineered "perfect-seed-only" configuration 295 (Fig. 7B). This result further indicates that without 3' critical non-seed pairing, "perfect-seed-only" 296 pairing is insufficient to support fully functional repression of lin-41.

We also asked whether 3' non-seed pairing could be sufficient in the absence of seed pairing.

298 We used the previously reported mutation lin-41(xe11) which creates an additional seed 299 mismatch to let-7a (Fig. 6F) (Ecsedi et al., 2015). We confirmed that lin-41(xe11) exhibits strong 300 vulva integrity defects and retarded heterochronic phenotypes (Fig. 6F), indicating that seed 301 pairing of lin-41 let-7a is not functionally dispensable, even in the context of extensive 3' pairing. 
A six-nucleotide critical supplementary pairing region of let-7a, involving g11-g16.

It has been shown that the 3' non-seed region of miRNAs can interact with targets and that

The functional architecture of base pairing configurations is particularly interesting for miRNAs with evolutionarily conserved 3' non-seed sequences (i.e., let-7a), and for evolutionarily conserved miRNA-target interactions (i.e., lin-41::let-7a). In this study, we utilized CRISPR/Cas9 genome editing for a systematic genetic investigation of the in vivo function of individual 3' nonseed nucleotide of let-7a in the regulation of lin-41 and other targets in C. elegans.

One noteworthy finding from our studies is that single nucleotide mutations at g11-g16 dramatically reduced let-7a in vivo function, suggesting their critical contribution to targeting.

316 Previous biochemical analysis and co-crystallized structure of human miRISC-target complex 317 have shown that miRNA can form helix with target at g13-g16, whilst g11 and g12 are structurally 318 hindered from duplexing with target (Grimson et al., 2007; Sheu-Gruttadauria et al., 2019b). In 319 contrast, our results indicate that g11 and g12 of let-7a should also duplex with complementary 320 targets. This expanded 3' pairing region (g11-g16) of let-7a suggests that miRISC can adopt a 321 previously unanticipated conformation that enables extended 3' pairing from g11 to g16.

Structural analyses of miRISC-target complexes reveal that duplexing of the miRNA seed to 323 target triggers conformational changes of AGO that then enable 3' pairing (Sheu-Gruttadauria et 324 al., 2019b). We note that the structural studies thus far have been for instances of perfect seed 325 pairing. By contrast, for the evolutionarily conserved let-7a target Trim71/lin-41, the LCSs are 
characterized by imperfect seed pairing accompanied by 3' pairing involving g11 and g12 in many species, and in some instances the specific configuration of seed mismatches is conserved, for example the g6:t6-GU pair and t5-bulge across 24 Caenorhabditis species (Nelson and Ambros, 2021). We suggest that these mismatched seed configurations may promote the AGO conformation that favors the pairing of $\mathrm{g} 11$ and $\mathrm{g} 12$, in addition to the previously identified 3' pairing region at g13-g16. Meanwhile, it has been shown that the miRISC conformation can also be affected by certain 3' pairing configurations. For example, an alternative AGO conformation can be induced when 3' pairing extends to the 3' end of the miRNA in the context of TDMD (Sheu-

334 Gruttadauria et al., 2019a). We thus suggest that at least for the let-7a::lin-41 interaction, miRISC may adopt a characteristic novel conformation that reflects simultaneous accommodation of both the imperfect seed pairing and the extended 3' helix.

The necessity of non-seed pairing for target repression efficacy.

It has long been recognized that perfect seed complementarity can be sufficient for miRISC to bind and repress target mRNAs (Brennecke et al., 2005; Doench and Sharp, 2004; Wee et al., 340 2012), and therefore 3' pairing should be required only to provide compensatory binding in cases 341 of weak seed pairing. However, studies of binding kinetics indicate that 3' pairing can enable 342 miRNA isoforms with identical perfect seed pairing to compete for target recognition (Xiao and 343 MacRae, 2020), highlighting the roles of 3' pairing in addition to the compensatory effect.

In our study, we find that for lin-41 LCS mutations that enable perfect seed complementarity to let-7a, the WT 3' non-seed pairing is still necessary for let-7a to confer functional repression of lin-41 (Fig. 6). Similarly, we show that let-7a family paralogs can confer only a modest degree of 347 repression of lin-41 via engineered perfect seed pairing (Fig. 7). These findings run counter to the 348 assumption that perfect seed pairing should be sufficient for miRISC to confer repression, and 349 provide an example of where 3' non-seed pairing is biologically required for the efficacy of an 350 evolutionarily conserved miRNA-target interaction. Moreover, among the predicted sites with let- 
$7 a$ critical non-seed pairing, all the sites in daf- 12 and 4 out of 7 sites in $h b l-1$ have perfect seed pairing (Fig. S6); however, daf-12 and hbl-1 are nevertheless de-repressed in the let-7ag13 nonseed mutant, supporting the conclusion that non-seed pairing can be critical even for sites with perfect seed complementarity.

Currently, we can only speculate about the mechanistic basis for this requirement for 3' pairing in the context of perfect seed match: perhaps certain structural peculiarities of the 3' UTRs render let-7a "seed-only" pairing kinetically unfavorable, or perhaps repression of lin-41 by let-7a requires the recruitment of cofactors that recognize certain miRISC conformational features induced by g11-g16 pairing.

\section{Contribution of the $3^{\prime}$ non-seed pairing to the target specificity of let-7 family paralogs.}

Recent reports showed that the $C$. elegans let-7a family paralog miR-48, which does not regulate lin-41 in WT, can substitute for let-7a in repressing lin-41 if the lin-41 LCSs were replaced with a site with a perfect seed match plus extensive 3' pairing to miR-48 (Broughton et al., 2016). It was proposed that the weak seed pairing of let-7a to lin-41 necessitates the 3' pairing, which differs remarkably between let-7a and $m i R-48$, and consequently determines the targeting specificity whereby lin-41 is regulated by let-7a, not miR-48 (Brancati and Grosshans, 2018). Thus,

371 reinforcement of the previous model of specificity determination since: (1) in our study, miR-84 is

372 expressed at levels and with temporal profile identical to WT let-7a; (2) unlike miR-48, which 373 differs from let-7a at every non-seed position, miR-84 has only 5 nucleotides different from let-7a. 
We also show that the in vivo functional distinction between let-7a and miR-84 primarily reflects the specificity of let-7a for targeting lin-41, in addition to other targets that require 3' nonseed pairing (Fig. S5). Interestingly, in $C$. elegans, $h b l-1$ is synergistically repressed by let-7a

377 paralogs at L2-to-L3 transition, and by let-7a at L4-to-adult transition (Abrahante et al., 2003;

378 Grosshans et al., 2005; Ilbay and Ambros, 2019). Notably, hbl-1 3' UTR contains sites expected

379 to be specifically recognized by let-7a (through imperfect seed +3 '-sup), and sites that can be

380 recognized by any paralog in let-7 family (through perfect seed) (Fig. S6D). Since we found that 381 functional repression of $\mathrm{hbl}$-1 in L4 stage requires 3' non-seed pairing (Fig. 5), the “imperfect seed $382+3$ '-sup" sites may enable the let-7a-specific repression of $h b l-1$ in particular tissues and at late 383 larval stages when let-7a is expressed, while the sites with perfect seed pairing would presumably 384 mediate repression of $h$ hl- 1 by let-7 family paralogs at earlier larval stages and/or in tissues devoid of let-7a expression.

\section{Multiplicity of C. elegans let-7a targets with 3' non-seed pairing.}

Our computational analysis identified 624 genes in C. elegans that contain 3' UTR sequences predicted to bind let-7a with 3' non-seed pairing. This finding is consistent with previous highthroughput analyses of miRNA-target chimeric ligation products, which also identified multiple let-

390 7a targets with 3' non-seed complementarity (Broughton et al., 2016; Grosswendt et al., 2014).

391 By ribosome profiling, we confirmed that 3' non-seed pairing is critical for the let-7a repression of

392 at least 8 genes and that over-expression of daf-12 and $h b l-1$ contribute to the developmental 393 phenotypes of the let-7a g13 mutant (Fig. 5). This finding indicates that in addition to lin-41, at 394 least daf-12 and hbl-1 are also phenocritical let-7a targets in C. elegans (Ecsedi et al., 2015). We 395 did not test the phenotypic consequences of the other over-expressed let-7a 3'-sup targets, which 396 are not known to be involved in the heterochronic pathway. These genes could contribute to let397 7a phenotypes not assayed in this study. 
There are limitations to the use of ribosome profiling of whole animals for the confirmation of in vivo miRNA targeting. In particular, the data could contain numerous false negatives, especially

400 for broadly-expressed genes that are repressed by miRNAs in only specific tissues or cell types.

401 For example, in our analysis, although we identified eight target genes with 3' non-seed

402 complementarity that were upregulated in the let-7a g13 mutant, as a class, genes with such 403 complementarity were not statistically enriched (Fig. S5E). Similarly, potential let-7a target genes

404 with g18 pairing were also not statistically enriched among the de-repressed genes in let-7 g18 405 mutant (Fig. S2 and data not shown).

Constraints of the evolutionary conservation of let-7a sequence.

The entire let-7a sequence is almost perfectly conserved across bilaterian phyla (Fig.1) (Wolter et al., 2017), suggesting phylogenetically ubiquitous involvement of let-7a in interactions that constrain the g9-g22 sequence. Our results indicate that the conservation of g11-g16 of let-

$4107 a$ could be driven by their phenocritical roles in the repression of conserved targets (i.e., lin-

$41141 /$ Trim71). Importantly, sequence constraints by target pairing require a multiplicity of targets

412 with a given pairing configuration (John et al., 2004). Our finding of the multiplicity of let-7a targets

413 with critical non-seed pairing supports the hypothesis that the 3'-sup pairing could constrain the 414 sequence of $\mathrm{g} 11-\mathrm{g} 16$.

In contrast to the g11-g16 sequence, our data do not appreciably illuminate potential mechanisms for the conservation of let-7a 3' distal nucleotides (g17-g22), or the bridge 417 nucleotides (g9-g10). However, although single nucleotide or compound mutations were 418 phenotypically tolerated in g17-g22, we observed phenotypic effects of a g18 mutation in the 419 context of g16 mutation, suggesting involvement of 3' distal nucleotides in repressing targets with 420 obligate mismatches to g14-g16 to let-7a. It is also possible that let-7a 3' distal nucleotides could 421 interact with miRISC associated sequence-specific RNA binding proteins in certain contexts. 422 Meanwhile, mutations at g9-g10 were also phenotypically tolerated, consistent with the 
423 observation that let-7a is not predicted to engage in target recognition involving g9-g10 paring in

424 C. elegans, and that fully complementary target sites which involve the bridge pairing are rare in 425 vertebrates (Bartel, 2018). However, miRNA bridge nucleotides can be exposed on the miRISC 426 surface even when they are complementary to the target (Sheu-Gruttadauria et al., 2019a). We

427 thus hypothesize that g9-g10 of let-7a may be constrained by association with conserved RNA 428 binding proteins that recognize these nucleotides, perhaps in the context of let-7a biogenesis or 429 target binding.

430 It is also possible that the identity of g9-g10 or g17-g22 nucleotides of let-7a could be critical 431 for target repression under unconventional conditions (i.e., stress), and/or required for target 432 interactions unrelated to the phenotypes that we monitored. The latter possibility is supported by 433 our analysis that the g18 mutant displays molecular phenotypes by Ribo-seq and RNA-seq (Fig. 434 S3). Such molecular phenotyping of other distal 3' nucleotides of let-7a, and other 'silent' 435 nucleotides of other miRNAs, could reveal otherwise experimentally inaccessible functionalities. 


\section{Acknowledgment}

438 This research was supported by funding from NIH grants R01GM088365, R01GM034028 and 439 R35GM131741 (V.A.). Some C. elegans strains were provided by the CGC, which is funded by 440 NIH Office of Research Infrastructure Programs (P40 OD010440).

\section{Author Contribution:}

442 Conceptualization: Y.D, I.V-L, V.A; Methodology: Y.D, I.V-L., V.A.; Formal analysis: Y.D, I.V-L., 443 V.A.; Investigation: Y.D, I.V-L.; Resources: I.V-L., V.A.; Data curation: Y.D.; Writing - original draft:

444 Y.D.; Writing - review \& editing: Y.D., I.V-L., V.A.; Supervision: V.A.; Project administration: V.A.; 445 Funding acquisition: V.A. 
Figure 1. let-7a is deeply conserved at all nucleotides across bilaterian species. A.

447 Summary of let-7 family miRNAs across bilaterian phylogeny. Bar length, number of let-7 family

448 members; bar color, sequence distances to hsa-let-7a-5p. B. Nucleotide frequency of the let-7 449 family isoforms most similar to hsa-let-7a-5p across bilaterians.

450 Figure 2. The 3' non-seed sequence determines the functional specificity of let-7a among

451 its paralogs. A. Strategy of the miR-84 swap mutagenesis. The dot-bracket notations show the 452 pre-miRNA structures predicted by RNAfold (Denman, 1993). B-E. Developmental profiles of 453 miR-84 and let-7a miRNAs determined using Fireplex assays. Expression levels are calibrated 454 with synthetic miR-84 and let-7a oligos. F. Representative lineages and COL-19::GFP expression 455 patterns of V1-V4 and V6 seam cells in WT and let-7(ma341). G. Seam cell numbers of young 456 adults. H-I. Representative expression patterns of COL-19::GFP in adults. Scale bars, $100 \mu \mathrm{m}$. 457 J-K. DIC images of the vulva region of adults. Scale bars, $25 \mu \mathrm{m}$. L. Adult lethality for WT, let4587 (ma341), let-7(n2853, seed mutant), and let-7(ma393, null). Lethal phenotypes are categorized 459 as either with vulva bursting (severe) or egg laying defective (mild).

460 Figure 3. Contribution of single 3' non-seed nucleotides to the in vivo function of let-7a. A. 461 Alignment of the mutants' pre-miRNA sequences in the single nucleotide mutational screen. The 462 non-seed region is boxed. B. Small RNA sequencing reads (prepared from L4 larvae) that 463 mapped to WT or mutant let-7a sequences for each single mutant. The reads mapping to WT let$4647 a$ for strains carrying the mutations of g11-g16 include WT let-7a miRNA from the balancer $465 m n D p 1$. C. Phenotype penetrance of COL-19::GFP pattern (top), numbers of progeny (middle) 466 and lethality (bottom). Lethal phenotypes are categorized identically to Fig. 2L. Abnormal COL467 19::GFP patterns are categorized as no Hyp7 expression (severe) or faint Hyp7 expression (mild). 468 D-G. DIC images of the vulva region in adults of let-7(ma432)(D); let-7(ma449)(E); let-7(m435)(F) 469 and let-7(ma449ma435)(G) at $25^{\circ} \mathrm{C}$. Scale bars, $25 \mu \mathrm{m}$. H. Functional synergy between g18 470 region and the critical non-seed region. Labels are identical to $\mathbf{C}$ (bottom). G. Summary of the 
471 functional merits of let-7a 3' non-seed nucleotides. Colored circle, 3' non-seed nucleotide; red

472 proportion, average penetrance of the severe vulva integrity phenotype at all temperatures.

473 Figure 4. The let-7a critical non-seed nucleotides confer in vivo function by repressing

474 both lin-41 and additional 3'-sup targets. A. Pairing configurations between let-7a and lin-41

475 LCS1/2. B-E. DIC images of the adult vulva regions representative of lin-41(tn1541)(B), lin476 41(tn1541);let-7(ma432)(C), lin-41(tn1541ma480);let-7(ma432)(D) and lin-41(tn1541ma480)(E)

477 at $25^{\circ} \mathrm{C}$. Scale bars, $25 \mu \mathrm{m}$. F. Vulva integrity defects based on lethality (bottom) and the number 478 of progeny (top). Lethal phenotypes are categorized identically to Fig. 2L. G. Representative COL479 19::GFP pattern and penetrance of lin-41(tn1541ma480);let-7(ma432) young adults at $25{ }^{\circ} \mathrm{C}$. 480 Scale bars, $100 \mu \mathrm{m}$. H. Illustrative models proposing that both lin-41 and additional 3'-sup targets 481 are de-repressed in let-7a non-seed mutants.

482 Figure 5. let-7a represses a multiplicity of 3'-sup targets, including daf-12 and hbl-1, 483 through 3' non-seed pairing. A. Differential expression analysis of translatomes of lin484 41(tn1541ma480);let-7(ma432) and lin-41(tn1541). Volcano plot represents genes with increased 485 ribosome protected footprints (RPFs) in lin-41(tn1541ma480);let-7(ma432) compared to lin486 41(tn1541). Solid points, significantly increased genes (FC > 1.5, P.adj < 0.1 by DESeq2). Circled 487 points with text labels, genes with predicted let-7a 3'-sup sites; triangle points, genes with only 488 let-7a seed-only sites. B. Retarded COL-19::GFP patterns characteristic of lin489 41(tn1541ma480);let-7(ma432) young adults at $25^{\circ} \mathrm{C}$ under empty vector conditions (top), daf490 12(RNAi) (middle) or $h b l-1(R N A i)$ (bottom). Scale bars, $100 \mu \mathrm{m}$. C. Fold changes of mRNA 491 abundance and translational efficiency (TE), comparing lin-41(tn1541ma480);let-7(ma432) to lin492 41(tn1541). Red/orange points, genes with significantly increased mRNA abundance/TE (FC > 493 1.5, P.adj < 0.1 by DESeq2 for mRNA abundance or $\mathrm{P}<0.1$ by t-test for TE). Purple points, genes 494 with both significantly increased TE and mRNA abundance. Circled points, genes with 
495 significantly increased RPFs in (A). Predicted let-7a 3'-sup targets are text-labeled. Data in A and

496 C were generated by 3 biological replicas.

497 Figure 6. Both the critical non-seed and seed paring are functionally critical for let-7a in

498 repressing lin-41. A-F. Pairing configurations of let-7a to the lin-41 LCSs, and associated

499 phenotypes for lin-41(tn1541ma501) (A); lin-41(tn1541ma501ma480) (B), lin-41(ma501ma545)

500 (C), lin-41(tn1541) (D), lin-41(tn1541ma480) (E), and lin-41(ex11) (F), arranged in a matrixed

501 panel based on mismatch numbers in seed (vertical axis) and critical non-seed region (horizontal

502 axis). For each panel, phenotypes include major COL-19:GFP pattern and penetrance in adults

503 at $25^{\circ} \mathrm{C}$ (middle) and vulva defects (bottom) by adult lethality (bar charts) and numbers of progeny

504 (violin plots). Scale bars, $100 \mu \mathrm{m}$. Lethal phenotypes are categorized identically to Fig. $2 \mathrm{~L}$.

505 Figure 7. Perfect seed pairing alone is insufficient for let-7a family paralogs to confer full

506 repression of lin-41. A. Predicted pairing configurations of let-7a and family paralogs miR-

$50748 / 84 / 241$ to LCS1 (top) and LCS2 (bottom) of lin-41(ma501) transcribed from RNAhybird

508 (Rehmsmeier et al., 2004). B. Vulva integrity defects based on adult lethality (top) and number of

509 progeny (bottom) in lin-41(tn1541), lin-41(tn1541);let-7(ma393,null) and lin-41(tn1541ma501);let-

5107 (ma393). C. Schematic diagrams of predicted targeting configurations. 


\section{Methods}

\section{Phylogeny and conservation analysis}

Precursor and mature miRNA sequences, and a phylogenetic tree of metazoa species were downloaded from miRbase (v22.1) (Kozomara et al., 2019). An in-house code was developed to identify miRNAs that belong to the let-7 family based on the presence of the seed sequence "GAGGUA" at g2-g7 on the mature miRNA. For each species in the collection, the number of total miRNA loci and the number let-7 miRNA loci were counted. To calculate the distance of each member to the conserved hsa-let-7a, its sequence was aligned to the hsa-let-7a sequence and the number of mismatches in the alignment was counted. The phylogenetic trees were visualized with the program evolView v2 (He et al., 2016).

117 species encode let-7a family isoforms. From each species, the sequence of one, the most similar to let-7a, was extracted and used to construct a conservation profile. A similar analysis was done with the mir-1 and mir-34 families to obtain their conservation profiles.

\section{Targeted mutagenesis at the let-7a genomic locus}

CRISPR/Cas9 genome editing of let-7a locus was performed using the "jump board" strategy described in (Duan et al., 2020a) on strain VT3742, which carries the let-7(ma393) insertion of the "jump board" sequence in place of the pre-let-7 sequence, as well as the genetic balancer mnDp1(umnls25), and a transgene oxSi1091 expressing Cas9. Templates for dsDNA HR donors were prepared by cloning the wild type pre-let-7a and $500 \mathrm{bp}$ of flanking sequence into pCR2.1TOPO vector. The Q5 mutagenesis kit (NEB, Cat: E0554) was used to generate the mutant plasmids. Double-strand DNA donors were generated from the mutant plasmids by PCR with 73/106 base-pairs flanking the pre-let-7a, and the PCR product was purified by ethanol precipitation. Injection mixtures containing final concentrations of $30 \mathrm{ng} / \mu \mathrm{l}$ AltR_Cas9_crRNA_INPP4A_1/2 each, $10 \mathrm{ng} / \mu \mathrm{l}$ AltR_Cas-9_crRNA_dpy-10_cn64 as co-CRISPR marker stage. F1 dumpy animals were isolated and genotyped by PCR with let-7_SEQ_F5/R5 primers followed by an analysis of the PCR product using restriction digestion using EcoRV (NEB,

540 Cat:N3195) and Sanger sequencing as described in (Duan et al., 2020a). Mutants were 541 backcrossed with wild-type (N2 or VT1367) for at least two generations. 
543 Targeted mutagenesis at the lin-41 genomic locus

Ultramer single-strand DNA donors (lengths ranging from $115 \mathrm{nt}$ to $117 \mathrm{nt}$ ) with $35 \mathrm{nt}$ flanking homology were obtained from IDT. The injection mixture containing final concentrations of 25

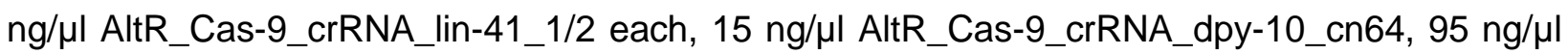
Alt-R tracrRNA (IDT, Cat:1072532), $325 \mathrm{ng} / \mu \mathrm{l}$ each ssDNA donor in $1 \mathrm{X}$ duplex buffer (IDT, Cat: 11010301) were incubated at room temperature for $10 \mathrm{~min}$ for pre-annealing and injected into the gonad of VT3867 or VT3873 which contain let-7(ma432) mutation, mnDp1(umn/s25) and transgene oxSi1091 expressing Cas9. F1 dumpy animals were isolated and genotyped by PCR with lin-41_SEQ_F2/R2 primers, followed by an analysis of the PCR product using Sanger sequencing. Mutants were backcrossed with N2 for at least two generations.

\section{Worm culturing and synchronization}

C.elegans were cultured on nematode growth medium (NGM) and fed with E. coli HB101 unless specified. To obtain populations of synchronized developing worms, gravid adults were collected and washed twice with water. Pellets of centrifuged worms were treated with $5 \mathrm{ml} 1 \mathrm{~N}$ $\mathrm{NaOH}$ and $1 \%(\mathrm{v} / \mathrm{v})$ sodium hypochlorite for $5 \mathrm{~min}$ with shaking to obtain embryos, and the embryos were rinsed with M9 buffer three times. The embryos were hatched in $10 \mathrm{ml} M 9$ buffer at $20^{\circ} \mathrm{C}$ for $16-18 \mathrm{hrs}$ with mild shaking. Hatched L1 larvae were transferred to plates at 30-50 worms per plate and replicate plates were cultured at $15^{\circ} \mathrm{C}, 20^{\circ} \mathrm{C}$, or $25^{\circ} \mathrm{C}$ for defined periods; samples of the population were examined by microscopy to confirm the developmental stage at 563 the time of harvest.

\section{Phenotypic assays for vulva defects}

566 The adult lethality characteristic of let-7(If), which results from rupture of the young adult animal 567 at the vulva, was scored after approximately $36 \mathrm{hrs}\left(15^{\circ} \mathrm{C}\right), 24 \mathrm{hrs}\left(20^{\circ} \mathrm{C}\right)$ or $16 \mathrm{hrs}\left(25^{\circ} \mathrm{C}\right)$ of 568 development (when at least $90 \%$ of the population had reached the adult stage). To score viable 569 progeny per adult, young adults were transferred to a fresh plate every $12 \mathrm{hrs}$ until those capable 570 of laying eggs had completed egg-laying. Only hatched eggs were counted. 


\section{Microscopy and heterochronic phenotypes}

573 Differential interference contrast and fluorescent images were obtained by Zeiss.Z1 equipped 574 with ZEISS Axiocam 503 camera. COL-19::GFP patterns were scored 10X objective. Adult alae 575 and GFP::LIN-41 images were obtained using a 100X objective. Lateral hypodermal 576 heterochronic cell lineage defects were scored by counting the number of seam cells per side of 577 each animal with the aid of the w/s51 transgenic seam cell reporter. Patterns of expression of the 578 adult-specific COL-19::GFP reporter were scored using the mals105 transgenic reporter. 579 Fluorescent images were processed by ImageJ FIJI (Schindelin et al., 2012).

\section{RNAi}

582 Overnight cultures of HT115 bacteria expressing dsRNA (Timmons et al., 2001) were transferred to LB broth and shaken at $37^{\circ} \mathrm{C}$ until $\mathrm{OD}_{600}$ was between $0.4-0.8$. The bacteria cultures were spread on NGM medium containing $100 \mu \mathrm{g} / \mathrm{ml}$ ampicillin and $1 \mathrm{mM} \mathrm{IPTG}$, and induced at room temperature for 24-48 hrs. HT115 bacteria strains for RNAi were obtained from the Ahringer library (Kamath et al., 2003).

\section{Total RNA preparation}

Harvested worms were washed with M9 medium, centrifuged, and the worm pellets were flashfrozen in liquid nitrogen. The worm pellets were thawed and lysed by adding 4X volumes of QIAzol (Qiagen, Cat: 79306) and shaking vigorously at room temperature for $15 \mathrm{~min}$. The total RNA was extracted by the addition of $0.85 \mathrm{X}$ volume chloroform, centrifugation, and recovery of the aqueous phase, which was then re-extracted with 1 volume phenol:chloroform:isoamyl alcohol (25:24:1, $\mathrm{pH}=5.5)$. Total RNA was then precipitated by add 1 volume of isopropanol and $0.5 \mu$ l GlycoBlue (Invitrogen, Cat: AM9516), followed by incubation at $-80^{\circ} \mathrm{C}$ for at least $30 \mathrm{~min}$, and recovery by centrifugation at $25,000 \mathrm{rcf}$ for $10 \mathrm{~min}$ at $4^{\circ} \mathrm{C}$. The supernatants were then removed, and the RNA

597 pellets were subsequently washed twice by $70 \%(\mathrm{v} / \mathrm{v})$ ethanol, dried in air for $5 \mathrm{~min}$, dissolved in 598 water, and stored at $-80^{\circ} \mathrm{C}$. 
Synchronized populations of developing worms were cultured at $20{ }^{\circ} \mathrm{C}$ and harvested at $8 \mathrm{hrs}$ (L1), 22 hrs (L2), 36 hrs (L3), 46 hrs (L4) and 58 hrs (adult) after feeding. Total RNA was extracted and miRNA levels of miR-84 and let-7a were quantified by FirePlex miRNA assay (Abcam) with customized C.elegans miRNA panel following the manufacturer's instructions. Guava easyCyte

$6058 \mathrm{HT}$ (Millipore) was used for readout. To normalize the quantification, synthetic RNA 606 oligonucleotides with sequences of $m i R-84$ and let-7a (IDT) were serially diluted and subjected to 607 FirePlex miRNA assay. Equal amounts of total RNA were used for all the samples and replicates. 608 The amounts of miRNA in experimental samples were calculated from the standard curve 609 generated from the serial dilution of respective synthetic RNA oligonucleotides.

\section{Small RNA sequencing}

612 Synchronized populations of developing worms were cultured at $20{ }^{\circ} \mathrm{C}$ and harvested at the 613 mid-late L4 stage (45 hrs after feeding). Total RNA was extracted as described above. The small 614 RNA sequencing libraries were constructed using QIAseq miRNA Library Kit (Qiagen, Cat:331505 $615 \& 331595)$, and sequenced by Illumina NextSeq 500 system. The adaptor sequences were 616 trimmed from the 3' end of the raw reads by Cutadapt/1.9 using default parameters (Martin, 2011). 617 To quantify the wild type or mutant let-7a miRNAs, the trimmed reads were mapped with 618 Bowtie2/2.3.4.3 to either wild type or mutant let-7a sequences indexed with $-c$ using parameters --end-to-end -N 0 --no-1mm-upfront -L 22 (Langmead and Salzberg, 2012). To quantify the total small RNA reads, trimmed reads were size filtered by Cutadapt/1.9, and reads with a length between 20-25 bp were kept. The filtered reads were initially mapped with Bowtie2/2.3.4.3 to $C$. elegans rRNA and tRNA sequences from WBCel235 with default parameters to remove tRNA and rRNA fragments (Chan and Lowe, 2009, 2016), and remaining reads were mapped to $C$. elegans genome WBCel235 by Bowtie2/2.3.4.3 with default parameters. The numbers of reads that mapped uniquely to the genome were used to calculate the RPM of wild type and mutant let$7 a$.

\section{Ribosome Profiling}

630 Synchronized populations of developing worms were cultured at $20^{\circ} \mathrm{C}$ for $45 \mathrm{hrs}$ after feeding. 631 Harvested worms were washed with water three times and incubated at room temperature for 10 
$632 \mathrm{~min}$ to allow digestion of intestinal bacteria. Worms were then pelleted by centrifuge at 4,500 rcf

633 for 2 min at room temperature and residual water was removed until the total volumes were twice

634 as the worm pellets. The samples were then flashed frozen by liquid nitrogen and stored at $-80^{\circ} \mathrm{C}$.

\section{Monosome preparation}

636 Concentrated lysis buffer was add to each frozen sample to final concentration of $20 \mathrm{mM}$ Tris$637 \mathrm{HCl}(\mathrm{pH}=7.4), 150 \mathrm{mM} \mathrm{NaCl}, 5 \mathrm{mM} \mathrm{MgCl}_{2}, 0.5 X$ Protease Inhibitor (Sigma, Cat:P2714), $1 \mathrm{mM}$

638 DTT, $0.1 \mathrm{mg} / \mathrm{ml}$ cycloheximide (Millipore, Cat:C4859), 1\% (v/v) Triton X100 and 5 U/ml Turbo 639 DNase (Invitrogen, Cat:AM2238) (Ingolia et al., 2012), and worm pellets were kept on ice until 640 fully thawed. Suspended worms were transferred to $400 \mu \mathrm{m}$ silica beads tube (OPS Diagnostic, 641 Cat: PFAW-400-100-04) and lysed in bead beater homogenizer for 3 min at $4{ }^{\circ} \mathrm{C}$. Lysates were 642 then centrifuged at $25,000 \mathrm{rcf}$ for $10 \mathrm{~min}$ at $4{ }^{\circ} \mathrm{C}$, and supernatants were collected. To generate 643 monosomes, RNase I (Invitrogen, Cat: AM2294) was added to a final concentration of $0.2 \mathrm{U}$ per

$644 \mu \mathrm{l}$ of harvested worm pellet. The digestion was incubated at room temperature for $40 \mathrm{~min}$ with 645 gentle rotation and then quenched by adding SUPERase RNase inhibitor (Invitrogen, Cat: $646 \mathrm{AM} 2694)$ at $4 \mathrm{U}$ per RNase I unit. The lysates were then loaded onto 5-40 \% (m/v) sucrose 647 gradients prepared with lysis buffer without Triton X100 and centrifuged at 32,000 rpm for $3 \mathrm{hrs}$ 648 at $4^{\circ} \mathrm{C}$ in an SW41Ti rotor (Beckman Coulter, Cat:331362). The sucrose gradients were 649 fractionated using BR-188 Density Gradient Fractionation System with 60\% (m/v) sucrose as 650 chase solution, and monosome fractions were collected according to $\mathrm{OD}_{254}$ profiles.

3.5 X volumes of QIAzol reagent was added to the gradient fractions containing monosomes, and RNA was extracted and separated by $17.5 \%$ denaturing PAGE. A synthetic RNA 654 oligonucleotide with the length of $30 \mathrm{nt}$ was used as a size marker. The gel was stained by Sybr 655 Gold (Invitrogen, Cat: S11494) at room temperature for $10 \mathrm{~min}$, and a gel slice containing RNA of 656 approximately 30 nt was excised and ground by an RNase-free pellet pestle (Fisher Scientific, 657 Cat: 12-141-364). RNA was extracted from the gel slice by adding $500 \mu \mathrm{l}$ of $300 \mathrm{mM} \mathrm{NaAc}(\mathrm{pH}=$ 658 5.5), $1 \mathrm{mM}$ EDTA, and $0.25 \%(\mathrm{~m} / \mathrm{v})$ SDS and mildly shaking overnight at room temperature 659 (Ingolia et al., 2012). The gel granules were excluded using Spin-X tube filter (Millipore, Cat: 660 CLS8160) and the RNA was then concentrated by ethanol precipitation, dissolved in water, and 661 stored at $-80^{\circ} \mathrm{C}$. 5' phosphorylation and 3' dephosphorylation were performed with T4 PNK (NEB, 662 Cat: M0201S) following the manufacturer's instructions, and the products were subjected to 663 phenol/chloroform extraction and ethanol precipitation. cDNA libraries were constructed using 
664 QIAseq miRNA Library Kit (Qiagen, Cat:331505 \& 331595) following the manufacturer's 665 instructions, except that the amplifying PCR was conducted with 9-12 cycles, and sequencing 666 was perform using Illumina NextSeq 500 system.

\section{RPF data analysis}

The adaptor sequences were trimmed from the 3' end of the raw reads by the Cutadapt/1.9 software using default parameters (Martin, 2011). Reads were sized filtered to keep only reads with a length between 26-34 bp (a range that fits ribosome-protected fragments) (Aeschimann et al., 2015). The rRNA and tRNA reads were removed by initially mapping with Bowtie2/2.3.4.3 to C. elegans rRNA and tRNA sequences from WBCel235 with default parameters (Chan and Lowe,

673 2009, 2016; Langmead and Salzberg, 2012), and the remaining reads were mapped to the $C$. 674 elegans genome WBcel235 by Star/2.5.3 with default parameters (Dobin et al., 2013). The p675 offset of the 5 ' end of the mapped reads and the monosome periodicity were determined by 676 plastid/0.4.8, and gene counting on exons was generated by plastid_cs/0.4.8 with p-offset 677 adjusted (Dunn and Weissman, 2016; Santos et al., 2019). Differential expression analysis was 678 performed by DESeq2 with default parameters (Kucukural et al., 2019; Love et al., 2014). Genes 679 with max raw count smaller than 10 were excluded from the analysis. Volcano plots were 680 generated using ggplot2 (Hadley, 2016).

\section{mRNA enrichment by C.elegans specific ribosome RNA depletion}

683 Worm samples for RNA-seq were aliquoted from the ribosome profiling harvests before the 684 lysis step and frozen separately. The total RNA was extracted as described above. To enrich for 685 mRNA, rRNA was depleted as described in detail elsewhere (Duan et al., 2020b). rRNA-depleted 686 mRNA samples were then purified by RNA Clean \& Concentrator-5 Kit (ZYMO, Cat: R1015) 687 (Zhang et al., 2012). The RNA-seq libraries were constructed by NEBNext Ultra II RNA Library 688 Prep kit (NEB, Cat: E7775, E7335, E7500) and sequenced by Illumina NextSeq 500 system 689 (Duan et al., 2020b).

\section{RNA-seq and translational efficiency data analysis}

692 The adaptor sequences were trimmed from RNA-seq data and reads shorter than 15 nt were 693 filtered out from the analysis by Cutadapt/1.9. tRNA, signal recognition particle RNA (srpR), and 694 residual cytoplasmic rRNA reads were removed by initial mapping with Bowtie2/2.3.4.3, and the 
remaining reads were mapped to $C$. elegans genome WBcel235 by Star/2.5.3 with default parameters. Gene counting was done by featureCounts (Liao et al., 2014). Differential expression analysis was performed by DESeq2 with default settings (Kucukural et al., 2019; Love et al., 2014). To calculate the translational efficiency (TE), the genes with max raw count smaller than 10 for either RNA-seq or ribosome profiling were excluded from the analysis. The TE was calculated by dividing normalized ribosome profiling counts by normalized RNA-seq counts for each replica. Significance was calculated by the Student t-test. Volcano plots were generated using ggplot2.

\section{Target prediction}

3' UTR sequences of C.elegans genes were downloaded from WormBase Parasite website (Howe et al., 2016; Howe et al., 2017) for the genomic version WBcel235. The UTRs were sorted and filtered to remove redundant sequences. The final dataset contained 15058 3'UTR sequences for 13975 genes (some genes have more than one 3'UTR isoform). The target sites of let-7a sequence that obey one of the following criteria were predicted using the algorithm developed in (Veksler-Lublinsky et al., 2010) (1) perfect Watson-Crick complementarity (perfect match) to positions 2-7; (2) perfect match to positions 2-7 allowing 1 target bulge on positions 57; (3) perfect match to positions 2-8 allowing $1 \mathrm{GU} /$ Mismatch (MM) on positions 5-8; (4) perfect match to positions 2-7 allowing 1 mRNA bulge on positions 2-4; (5) a match to positions 2-8 allowing $1 \mathrm{GU} / \mathrm{MM}$ on positions 2-4.

714 For sites that obey the criteria, a flanking region of additional 20 nucleotides after the seed was extracted. The interaction duplex between the full site and the miRNA was then calculated using

716 RNAduplex (Lorenz et al., 2011). The duplex was parsed to identify both the seed type and the 717 non-seed type for each reported interaction as follows: (a) Seed type: (1) 2-7 full match ; (2) 2-7 $718+1$ target bulge on 5-7 or 2-8 with 1 GU/MM 5-8; (3) 2-7 +1 target bulge on 2-4 or 2-8 with 1 719 GU/MM 2-4. (b) NonSeedType: (1) perfect match to positions 11-13 or 12-14 or 13-16; (2) match 720 allowing GUs to positions $11-13$ or $12-14$ or $13-16 ;(-1)$ none of the above.

721 For genes that have multiple UTR sequences in the analysis, UTRs with redundant duplexes 722 were filtered out from the final report.

\section{Quantification and statistical analysis}


725 All data containing statistical analysis represent the mean \pm standard deviation of at least three

726 replications. The $p$-values representation is as follow: $0.05-0.01\left(^{*}\right) ; 0.01-0.001\left(^{* *}\right) ; 0.001-$

$7270.0001\left(^{* * *}\right) ;<0.0001\left(^{* * *}\right)$. Significance tests were conducted with Prism 8 if not mentioned. 
Abbott, A.L., Alvarez-Saavedra, E., Miska, E.A., Lau, N.C., Bartel, D.P., Horvitz, H.R., and Ambros, V. (2005). The let-7 MicroRNA family members mir-48, mir-84, and mir-241 function together to regulate developmental timing in Caenorhabditis elegans. Developmental cell 9, 403414.

Abrahante, J.E., Daul, A.L., Li, M., Volk, M.L., Tennessen, J.M., Miller, E.A., and Rougvie, A.E. (2003). The Caenorhabditis elegans hunchback-like gene lin-57/hbl-1 controls developmental time and is regulated by microRNAs. Developmental cell 4, 625-637. transition to adulthood through a single primary and four secondary targets. Life Sci Alliance 2. wide measurement of ribosomal occupancy by ribosome profiling. Methods $85,75-89$. essential for development or viability. Current biology : CB 20,367-373. Ambros, V. (2004). The functions of animal microRNAs. Nature 431, 350-355. Marker-Free Recovery of Custom Genetic Modifications with CRISPR/Cas9 in Caenorhabditis elegans. Genetics 198, 837-846.

746 Bagga, S., Bracht, J., Hunter, S., Massirer, K., Holtz, J., Eachus, R., and Pasquinelli, A.E. (2005).

747 Regulation by let-7 and lin-4 miRNAs results in target mRNA degradation. Cell 122, 553-563.

748 Balzeau, J., Menezes, M.R., Cao, S., and Hagan, J.P. (2017). The LIN28/let-7 Pathway in Cancer.

749 Frontiers in genetics 8, 31.

750 Bartel, D.P. (2018). Metazoan MicroRNAs. Cell 173, 20-51.

751 Bazzini, A.A., Lee, M.T., and Giraldez, A.J. (2012). Ribosome profiling shows that miR-430 752 reduces translation before causing mRNA decay in zebrafish. Science 336, 233-237.

753 Brancati, G., and Grosshans, H. (2018). An interplay of miRNA abundance and target site 754 architecture determines miRNA activity and specificity. Nucleic acids research 46, 3259-3269.

755 Brennecke, J., Stark, A., Russell, R.B., and Cohen, S.M. (2005). Principles of microRNA-target 756 recognition. PLoS biology 3, e85.

757 Brenner, J.L., Kemp, B.J., and Abbott, A.L. (2012). The mir-51 family of microRNAs functions in 758 diverse regulatory pathways in Caenorhabditis elegans. PloS one 7, e37185.

759 Broughton, J.P., Lovci, M.T., Huang, J.L., Yeo, G.W., and Pasquinelli, A.E. (2016). Pairing beyond 760 the Seed Supports MicroRNA Targeting Specificity. Molecular cell 64, 320-333. 
761 Chan, P.P., and Lowe, T.M. (2009). GtRNAdb: a database of transfer RNA genes detected in 762 genomic sequence. Nucleic acids research 37, D93-97.

763 Chan, P.P., and Lowe, T.M. (2016). GtRNAdb 2.0: an expanded database of transfer RNA genes 764 identified in complete and draft genomes. Nucleic acids research 44, D184-189.

765 Chi, S.W., Hannon, G.J., and Darnell, R.B. (2012). An alternative mode of microRNA target 766 recognition. Nature structural \& molecular biology 19, 321-327.

767 Denman, R.B. (1993). Using RNAFOLD to predict the activity of small catalytic RNAs. 768 BioTechniques 15, 1090-1095.

769 Djuranovic, S., Nahvi, A., and Green, R. (2012). miRNA-mediated gene silencing by translational 770 repression followed by mRNA deadenylation and decay. Science 336, 237-240.

771 Dobin, A., Davis, C.A., Schlesinger, F., Drenkow, J., Zaleski, C., Jha, S., Batut, P., Chaisson, M., 772 and Gingeras, T.R. (2013). STAR: ultrafast universal RNA-seq aligner. Bioinformatics 29, 15-21.

773 Doench, J.G., and Sharp, P.A. (2004). Specificity of microRNA target selection in translational 774 repression. Genes \& development 18, 504-511.

775 Duan, Y., Choi, S., Nelson, C., and Ambros, V. (2020a). Engineering essential genes with a "jump 776 board" strategy using CRISPR/Cas9. MicroPubl Biol 2020.

777 Duan, Y., Sun, Y., and Ambros, V. (2020b). RNA-seq with RNase H-based ribosomal RNA 778 depletion specifically designed for C. elegans. MicroPubl Biol 2020.

779 Dunn, J.G., and Weissman, J.S. (2016). Plastid: nucleotide-resolution analysis of next-generation 780 sequencing and genomics data. BMC genomics 17, 958.

781 Ecsedi, M., and Grosshans, H. (2013). LIN-41/TRIM71: emancipation of a miRNA target. Genes 782 \& development 27, 581-589.

783 Ecsedi, M., Rausch, M., and Grosshans, H. (2015). The let-7 microRNA directs vulval 784 development through a single target. Developmental cell 32, 335-344.

785 Friedman, R.C., Farh, K.K., Burge, C.B., and Bartel, D.P. (2009). Most mammalian mRNAs are 786 conserved targets of microRNAs. Genome research 19, 92-105.

787 Giraldez, A.J., Mishima, Y., Rihel, J., Grocock, R.J., Van Dongen, S., Inoue, K., Enright, A.J., and 788 Schier, A.F. (2006). Zebrafish MiR-430 promotes deadenylation and clearance of maternal 789 mRNAs. Science 312, 75-79.

790 Grimson, A., Farh, K.K., Johnston, W.K., Garrett-Engele, P., Lim, L.P., and Bartel, D.P. (2007). 791 MicroRNA targeting specificity in mammals: determinants beyond seed pairing. Molecular cell 27, 792 91-105. 
793 Grosshans, H., Johnson, T., Reinert, K.L., Gerstein, M., and Slack, F.J. (2005). The temporal 794 patterning microRNA let-7 regulates several transcription factors at the larval to adult transition in 795 C. elegans. Developmental cell 8, 321-330.

796 Grosswendt, S., Filipchyk, A., Manzano, M., Klironomos, F., Schilling, M., Herzog, M., Gottwein, 797 E., and Rajewsky, N. (2014). Unambiguous identification of miRNA:target site interactions by 798 different types of ligation reactions. Molecular cell 54, 1042-1054.

799 Hadley, W. (2016). Ggplot2 (New York, NY: Springer Science+Business Media, LLC).

800 He, Z., Zhang, H., Gao, S., Lercher, M.J., Chen, W.H., and Hu, S. (2016). Evolview v2: an online 801 visualization and management tool for customized and annotated phylogenetic trees. Nucleic 802 acids research 44, W236-241.

803 Helwak, A., Kudla, G., Dudnakova, T., and Tollervey, D. (2013). Mapping the human miRNA 804 interactome by CLASH reveals frequent noncanonical binding. Cell 153, 654-665.

805 Hertel, J., Bartschat, S., Wintsche, A., Otto, C., Students of the Bioinformatics Computer, L., and 806 Stadler, P.F. (2012). Evolution of the let-7 microRNA family. RNA biology 9, 231-241.

807 Howe, K.L., Bolt, B.J., Cain, S., Chan, J., Chen, W.J., Davis, P., Done, J., Down, T., Gao, S., 808 Grove, C., et al. (2016). WormBase 2016: expanding to enable helminth genomic research. 809 Nucleic acids research 44, D774-780.

810 Howe, K.L., Bolt, B.J., Shafie, M., Kersey, P., and Berriman, M. (2017). WormBase ParaSite - a 811 comprehensive resource for helminth genomics. Mol Biochem Parasitol 215, 2-10.

812 Ilbay, O., and Ambros, V. (2019). Regulation of nuclear-cytoplasmic partitioning by the lin-28-lin81346 pathway reinforces microRNA repression of $\mathrm{HBL}-1$ to confer robust cell-fate progression in $\mathrm{C}$. 814 elegans. Development 146.

815 Ingolia, N.T. (2016). Ribosome Footprint Profiling of Translation throughout the Genome. Cell 165, 816 22-33.

817 Ingolia, N.T., Brar, G.A., Rouskin, S., McGeachy, A.M., and Weissman, J.S. (2012). The ribosome 818 profiling strategy for monitoring translation in vivo by deep sequencing of ribosome-protected 819 mRNA fragments. Nature protocols 7, 1534-1550.

820 John, B., Enright, A.J., Aravin, A., Tuschl, T., Sander, C., and Marks, D.S. (2004). Human 821 MicroRNA targets. PLoS biology 2, e363.

822 Kamath, R.S., Fraser, A.G., Dong, Y., Poulin, G., Durbin, R., Gotta, M., Kanapin, A., Le Bot, N., 823 Moreno, S., Sohrmann, M., et al. (2003). Systematic functional analysis of the Caenorhabditis 824 elegans genome using RNAi. Nature 421, 231-237.

825 Kozomara, A., Birgaoanu, M., and Griffiths-Jones, S. (2019). miRBase: from microRNA 826 sequences to function. Nucleic acids research 47, D155-D162. 
827 Kucukural, A., Yukselen, O., Ozata, D.M., Moore, M.J., and Garber, M. (2019). DEBrowser: 828 interactive differential expression analysis and visualization tool for count data. BMC genomics $82920,6$.

830 Lai, E.C. (2002). Micro RNAs are complementary to 3' UTR sequence motifs that mediate 831 negative post-transcriptional regulation. Nature genetics 30, 363-364.

832 Langmead, B., and Salzberg, S.L. (2012). Fast gapped-read alignment with Bowtie 2. Nature 833 methods 9, 357-359.

834 Lee, H., Han, S., Kwon, C.S., and Lee, D. (2016). Biogenesis and regulation of the let-7 miRNAs 835 and their functional implications. Protein Cell 7, 100-113.

836 Lee, R.C., Feinbaum, R.L., and Ambros, V. (1993). The C. elegans heterochronic gene lin-4 837 encodes small RNAs with antisense complementarity to lin-14. Cell 75, 843-854.

838 Lewis, B.P., Burge, C.B., and Bartel, D.P. (2005). Conserved seed pairing, often flanked by 839 adenosines, indicates that thousands of human genes are microRNA targets. Cell 120, 15-20.

840 Liao, Y., Smyth, G.K., and Shi, W. (2014). featureCounts: an efficient general purpose program 841 for assigning sequence reads to genomic features. Bioinformatics 30, 923-930.

842 Lim, L.P., Lau, N.C., Garrett-Engele, P., Grimson, A., Schelter, J.M., Castle, J., Bartel, D.P., 843 Linsley, P.S., and Johnson, J.M. (2005). Microarray analysis shows that some microRNAs 844 downregulate large numbers of target mRNAs. Nature 433, 769-773.

845 Lorenz, R., Bernhart, S.H., Honer Zu Siederdissen, C., Tafer, H., Flamm, C., Stadler, P.F., and 846 Hofacker, I.L. (2011). ViennaRNA Package 2.0. Algorithms Mol Biol 6, 26.

847 Love, M.I., Huber, W., and Anders, S. (2014). Moderated estimation of fold change and dispersion 848 for RNA-seq data with DESeq2. Genome biology 15, 550.

849 Martin, M. (2011). Cutadapt removes adapter sequences from high-throughput sequencing reads. $850201117,3$.

851 Nelson, C., and Ambros, V. (2019). Trans-splicing of the C. elegans let-7 primary transcript 852 developmentally regulates let-7 microRNA biogenesis and let-7 family microRNA activity. 853 Development 146.

854 Nelson, C., and Ambros, V. (2021). A cohort of Caenorhabditis species lacking the highly 855 conserved let-7 microRNA. G3 Genes|Genomes|Genetics.

856 Nottrott, S., Simard, M.J., and Richter, J.D. (2006). Human let-7a miRNA blocks protein 857 production on actively translating polyribosomes. Nature structural \& molecular biology $13,1108-$ 8581114. 
859 Pasquinelli, A.E., McCoy, A., Jimenez, E., Salo, E., Ruvkun, G., Martindale, M.Q., and Baguna, J. (2003). Expression of the 22 nucleotide let-7 heterochronic RNA throughout the Metazoa: a role in life history evolution? Evolution \& development 5, 372-378.

862 Paul, P., Chakraborty, A., Sarkar, D., Langthasa, M., Rahman, M., Bari, M., Singha, R.S., Malakar, 863 A.K., and Chakraborty, S. (2018). Interplay between miRNAs and human diseases. Journal of 864 cellular physiology 233, 2007-2018.

865 Pawlica, P., Sheu-Gruttadauria, J., MacRae, I.J., and Steitz, J.A. (2020). How Complementary 866 Targets Expose the microRNA 3' End for Tailing and Trimming during Target-Directed microRNA 867 Degradation. Cold Spring Harbor symposia on quantitative biology.

868 Rehmsmeier, M., Steffen, P., Hochsmann, M., and Giegerich, R. (2004). Fast and effective 869 prediction of microRNA/target duplexes. Rna 10, 1507-1517.

870 Reinhart, B.J., Slack, F.J., Basson, M., Pasquinelli, A.E., Bettinger, J.C., Rougvie, A.E., Horvitz, 871 H.R., and Ruvkun, G. (2000). The 21-nucleotide let-7 RNA regulates developmental timing in 872 Caenorhabditis elegans. Nature 403, 901-906.

873 Roush, S., and Slack, F.J. (2008). The let-7 family of microRNAs. Trends in cell biology 18, 505874516.

875 Salomon, W.E., Jolly, S.M., Moore, M.J., Zamore, P.D., and Serebrov, V. (2015). Single-Molecule Imaging Reveals that Argonaute Reshapes the Binding Properties of Its Nucleic Acid Guides. Cell 162, 84-95.

Santos, D.A., Shi, L., Tu, B.P., and Weissman, J.S. (2019). Cycloheximide can distort measurements of mRNA levels and translation efficiency. Nucleic acids research. Schindelin, J., Arganda-Carreras, I., Frise, E., Kaynig, V., Longair, M., Pietzsch, T., Preibisch, S., Rueden, C., Saalfeld, S., Schmid, B., et al. (2012). Fiji: an open-source platform for biologicalimage analysis. Nature methods 9, 676-682.

883 Schirle, N.T., Sheu-Gruttadauria, J., and MacRae, I.J. (2014). Structural basis for microRNA 884 targeting. Science 346, 608-613.

885 Sheu-Gruttadauria, J., Pawlica, P., Klum, S.M., Wang, S., Yario, T.A., Schirle Oakdale, N.T., 886 Steitz, J.A., and MacRae, I.J. (2019a). Structural Basis for Target-Directed MicroRNA 887 Degradation. Molecular cell 75, 1243-1255 e1247.

888 Sheu-Gruttadauria, J., Xiao, Y., Gebert, L.F., and MacRae, I.J. (2019b). Beyond the seed: 889 structural basis for supplementary microRNA targeting by human Argonaute2. The EMBO journal. 890 Sokol, N.S., Xu, P., Jan, Y.N., and Ambros, V. (2008). Drosophila let-7 microRNA is required for 891 remodeling of the neuromusculature during metamorphosis. Genes \& development 22, 15918921596. 
893 Spike, C.A., Coetzee, D., Eichten, C., Wang, X., Hansen, D., and Greenstein, D. (2014). The 894 TRIM-NHL protein LIN-41 and the OMA RNA-binding proteins antagonistically control the 895 prophase-to-metaphase transition and growth of Caenorhabditis elegans oocytes. Genetics 198, 896 1535-1558.

897 Sulston, J.E., Schierenberg, E., White, J.G., and Thomson, J.N. (1983). The embryonic cell 898 lineage of the nematode Caenorhabditis elegans. Developmental biology 100, 64-119.

899 Tennessen, J.M., and Thummel, C.S. (2008). Developmental timing: let-7 function conserved 900 through evolution. Current biology : CB 18, R707-708.

901 Timmons, L., Court, D.L., and Fire, A. (2001). Ingestion of bacterially expressed dsRNAs can 902 produce specific and potent genetic interference in Caenorhabditis elegans. Gene 263, 103-112. 903 Veksler-Lublinsky, I., Shemer-Avni, Y., Kedem, K., and Ziv-Ukelson, M. (2010). Gene bi-targeting 904 by viral and human miRNAs. BMC bioinformatics 11, 249.

905 Vella, M.C., Choi, E.Y., Lin, S.Y., Reinert, K., and Slack, F.J. (2004). The C. elegans microRNA 906 let-7 binds to imperfect let-7 complementary sites from the lin-41 3'UTR. Genes \& development 907 18, 132-137.

908 Wahlquist, C., Jeong, D., Rojas-Munoz, A., Kho, C., Lee, A., Mitsuyama, S., van Mil, A., Park, 909 W.J., Sluijter, J.P., Doevendans, P.A., et al. (2014). Inhibition of miR-25 improves cardiac 910 contractility in the failing heart. Nature 508, 531-535.

911 Wee, L.M., Flores-Jasso, C.F., Salomon, W.E., and Zamore, P.D. (2012). Argonaute divides its 912 RNA guide into domains with distinct functions and RNA-binding properties. Cell 151, 1055-1067. 913 Wolter, J.M., Le, H.H., Linse, A., Godlove, V.A., Nguyen, T.D., Kotagama, K., Lynch, A., Rawls, 914 A., and Mangone, M. (2017). Evolutionary patterns of metazoan microRNAs reveal targeting 915 principles in the let-7 and miR-10 families. Genome research 27, 53-63.

916 Worringer, K.A., Rand, T.A., Hayashi, Y., Sami, S., Takahashi, K., Tanabe, K., Narita, M., 917 Srivastava, D., and Yamanaka, S. (2014). The let-7/LIN-41 pathway regulates reprogramming to 918 human induced pluripotent stem cells by controlling expression of prodifferentiation genes. Cell 919 stem cell 14, 40-52.

920 Wu, L., Fan, J., and Belasco, J.G. (2006). MicroRNAs direct rapid deadenylation of mRNA. 921 Proceedings of the National Academy of Sciences of the United States of America 103, 40349224039.

923 Xiao, Y., and MacRae, I.J. (2020). Robust differential microRNA targeting driven by 924 supplementary interactions in vitro. Rna 26, 162-174.

925 Zhang, Z., Theurkauf, W.E., Weng, Z., and Zamore, P.D. (2012). Strand-specific libraries for high 926 throughput RNA sequencing (RNA-Seq) prepared without poly(A) selection. Silence 3, 9. 
bioRxiv preprint doi: https://doi.org/10.1101/2021.03.29.437276; this version posted March 29, 2021. The copyright holder for this preprint (which was not certified by peer review) is the author/funder. All rights reserved. No reuse allowed without permission. 
A

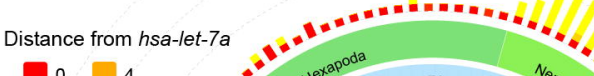

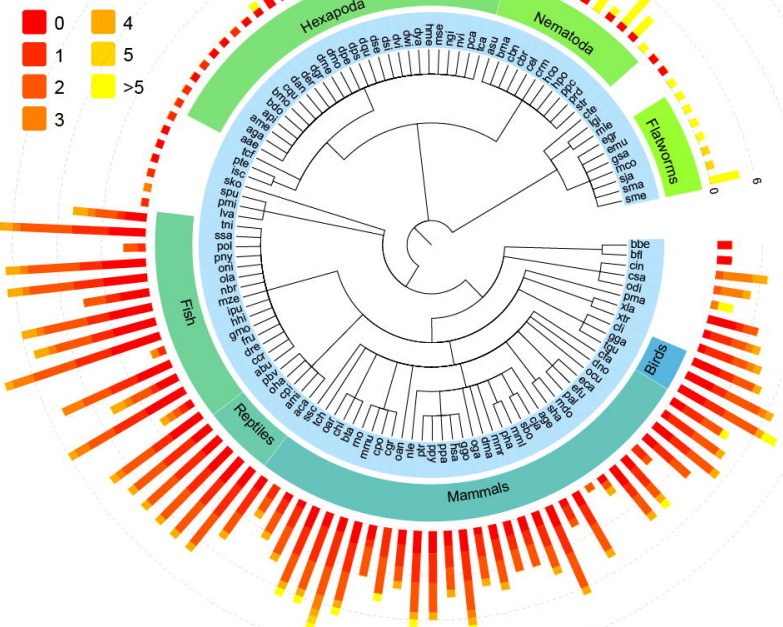

B
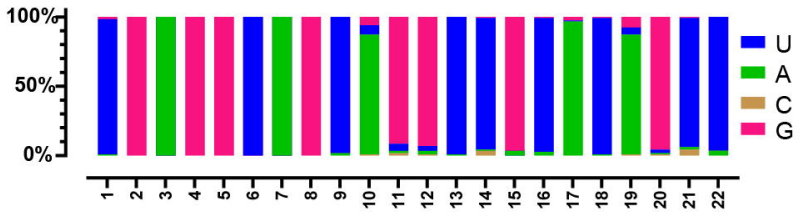

nucleotide from $\mathbf{5}^{\prime}$ end $\quad \mathrm{n}=117$ 
A let-7a

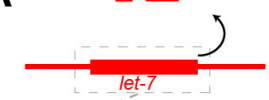

WT

\section{CRISPR/Cas9}

$\operatorname{miR}-84 \sim \sim$

\section{let-7(ma341)}

WT

UGAGGUAGUAGGUUGUAUAGUUUGGAAUAUUACCACCGGUGAACUAUGCAAUUUUCUACCUUAC

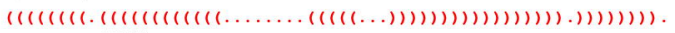
$\longmapsto$ miRNA $\longrightarrow$

$\longmapsto$ passenger—

let-7(ma341) UGAGGUAGUAUGUAAUAUUGUAUGGAAUAUUACCACCGGUGUACAAUGUUAUGUUCUACCUUAC

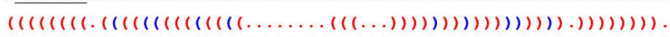

B WT
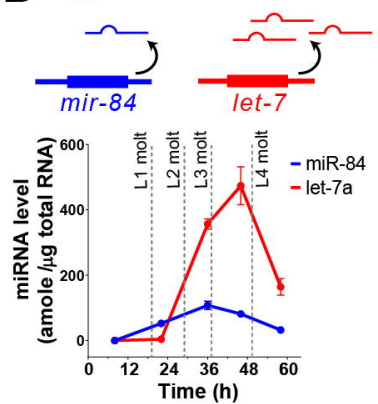

F
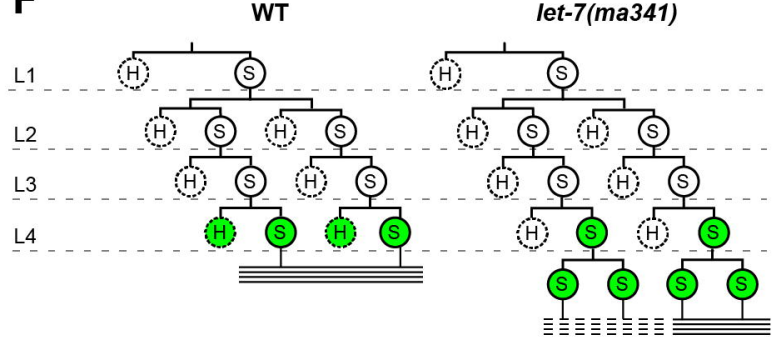

(H) Hyp7 (S) seam cell $\bigcirc$ COL-19:.GFP +

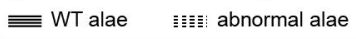

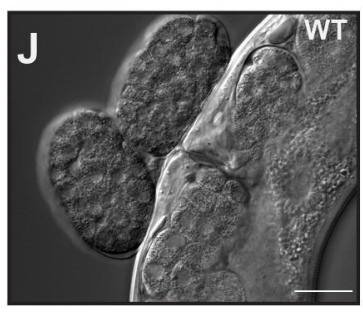

D mir-84(n4037)


G
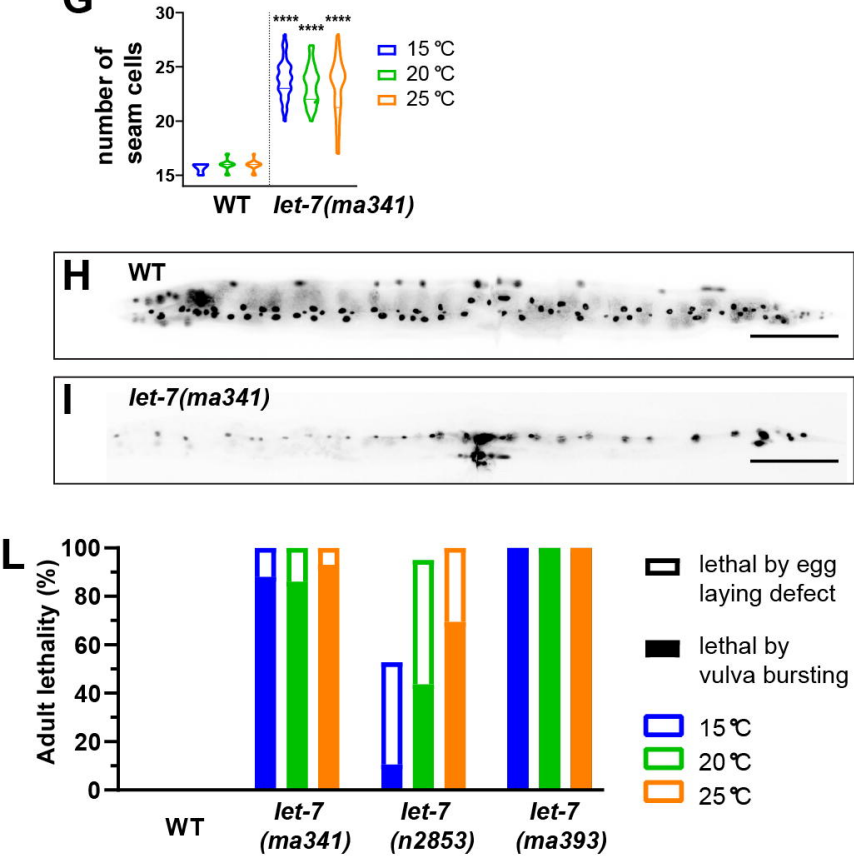
A lin-41(+);let-7(+)

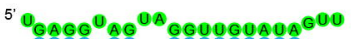

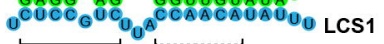
5 OAG GOAG OA GOOGOAOACOO

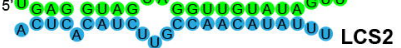

\section{lin-41(+);let-7(ma432)}

${ }^{5} \mathrm{O}_{\mathrm{GAGG}} \mathrm{AO}^{\mathrm{OA}} \mathrm{GG}^{\circ} \mathrm{OGOAOA} \mathrm{O}^{\mathrm{O} O 0}$

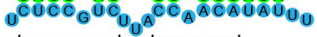

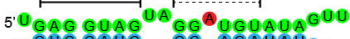

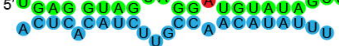

\section{lin-41(ma480);let-7(ma432)}

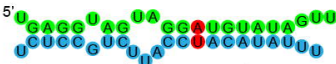

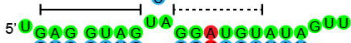
$A^{\text {COOC } \mathrm{CAOO}} \mathrm{O}_{0} \mathrm{O}^{\mathrm{COCAOCAOAO}}$

\section{lin-41(ma480);let-7(+)}

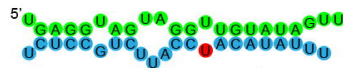

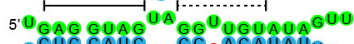

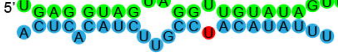

- mutation
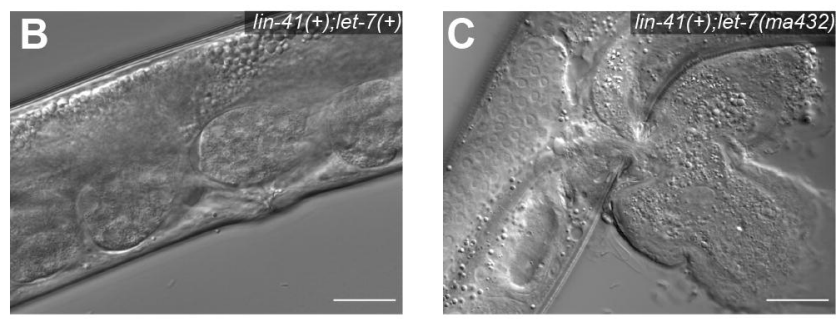

$\mathbf{F}$

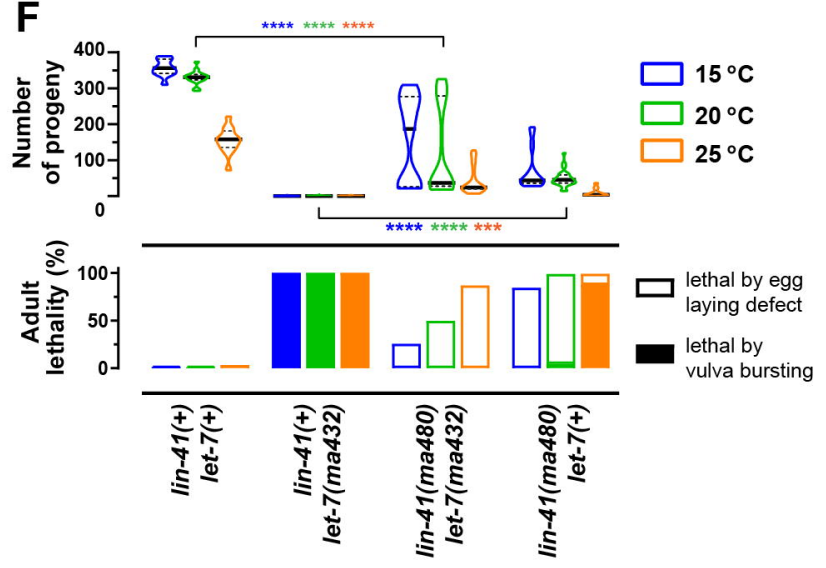

$\mathrm{H}$

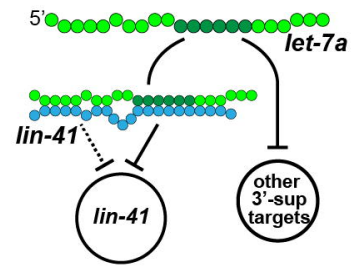

lin-41(+); let-7(ma432)

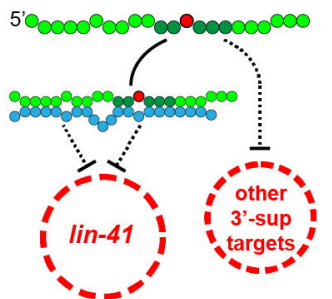



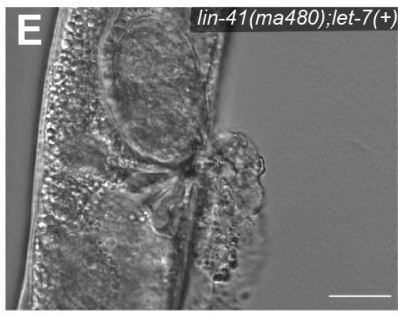

G

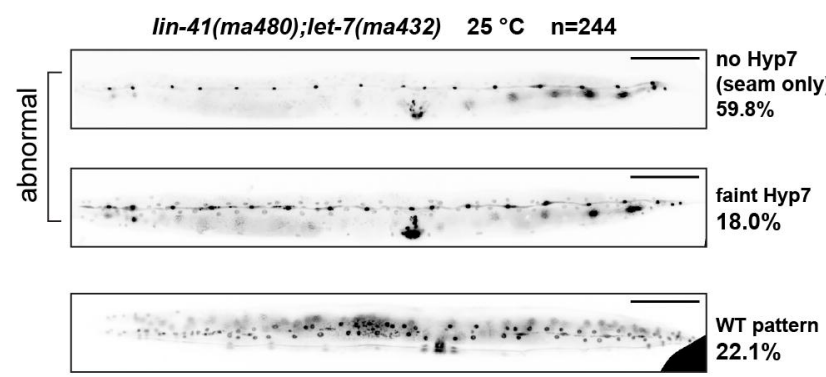

lin-41(ma480); let-7(ma432)

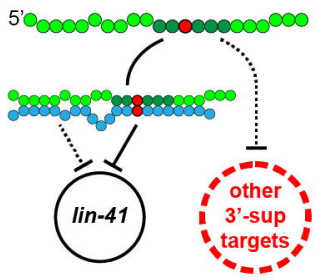

lin-41(ma480); let-7(+)

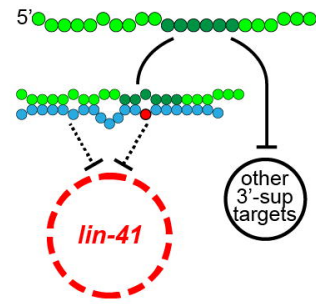




\section{Mismatches in critical non-seed pairing}

0

1

3

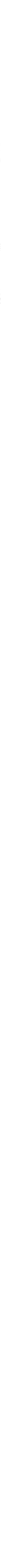




\section{$\mathbf{A}$} $5_{\text {UGAGGUAG }}^{\text {UA }}{ }_{\text {GGUUGUAUAG }}{ }^{\mathrm{UU}}$ let-7a ${ }_{*}^{A C U C C A U C}{ }_{\text {UUG }}$ CCAACAUAU $_{U_{U}}$

$U_{\text {GAGGUAG }}^{\text {UA }}{ }_{\text {GGUUGUAUAG }}{ }^{\text {UU }}$ $U_{*}^{C U C C A U C}$ UUA $_{\text {CCAACAUAU }}$

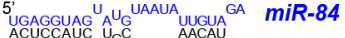
${ }_{A C U C C A U C} U_{G} C_{C} \ldots{ }_{A A C A U}$

$U_{\text {GAGGUAG }}$ AUG $^{\text {UAAUA }}$ UUGUA $\mathrm{U}_{*}^{\text {CUCCAUC }} \mathrm{UAC}_{\mathrm{C}} \ldots$ AACAU $_{\text {A }}$

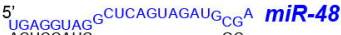
ACUCCAUC $U^{G C}{ }_{C}$

$U_{\text {GAGGUAG }}{ }^{\text {CUCA - }}$ GUA GAUGCGA $U^{\text {CUCCAUC }}$ UUACCAA $_{\text {UAU }}$ AUU

5' ACUCCAUC $_{*} \cup{ }_{\text {GC }}$ CAACAUAU

miR-241

GAGgUaG G ${ }^{C}{ }^{C}$ AGAAAUGA

$U_{*}^{\text {CUCCAUC }} U^{U A C} C_{\text {AACAUAUU }}$

* mutation in ma501

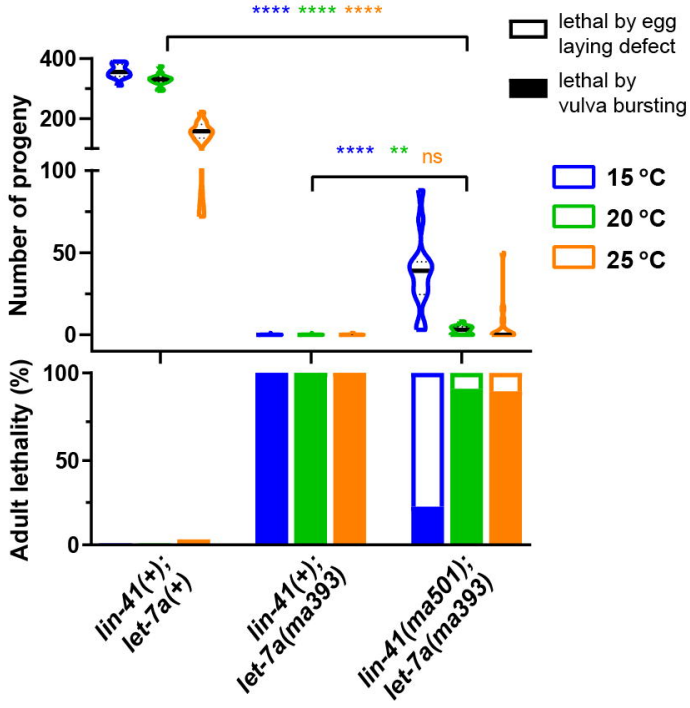

C Iin-41(+);let-7(+)

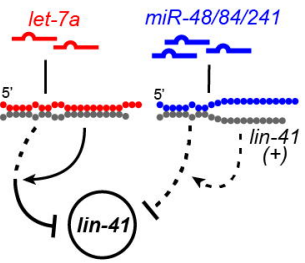

lin-41(+);let-7(ma393,nuII)

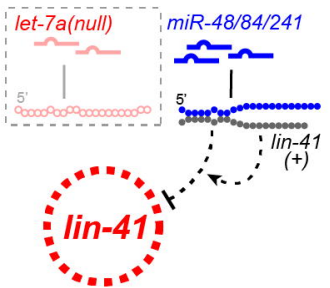

lin-41(ma501);let-7(ma393,nuIl)

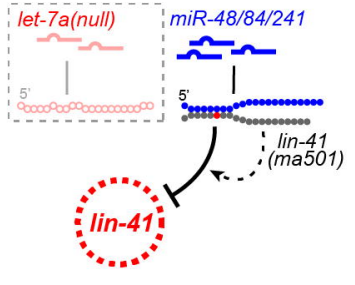

